\title{
Vogel-Kirsche (Prunus avium L.) - Baum des Jahres 2010
}

\author{
VeIt MARTIN Dorken
}

\begin{abstract}
Wild cherry (Prunus avium) is tree of the year 2010. Its biology, ecology and use are summarized.
\end{abstract}

\section{Zusammenfassung}

Die Vogel-Kirsche (Prunus avium) ist Baum des Jahres 2010. Ihre Biologie, Ökologie und ihr Nutzen werden vorgestellt.

\section{Einleitung}

Seit 1989 kürt das Kuratorium „Baum des Jahres“ jährlich einen Jahresbaum. Auswahlkriterien sind unter anderem Seltenheit, Bedrohung sowie ästhetische, ökologische und landschaftsgestalterische Aspekte. Für dieses Jahr fiel die Wahl auf die heimische Vogel- oder Süß-Kirsche (Prunus avium = Cerasus avium). Sie ist ein tierökologisch wertvoller Baum und prägt aufgrund ihrer auffälligen Blüte und der leuchtenden Herbstfärbung vielerorts die Landschaft. Zudem ist sie Stammform unserer Kultur-Süßkirsche.

\section{Systematik}

Die Vogel-Kirsche ist ein Vertreter der Rosengewächse (Rosaceae), die weltweit etwa 107 Gattungen mit rund 3100 Arten vereint (STÜTZEL 2005). Prunus umfasst rund 430 laubabwerfende sowie immergrüne Arten, die überwiegend in den gemäßigten Zonen der Nordhalbkugel heimisch sind (ROLOFF \& BÄRTELS 1996). Die weit bekannten und als Steinobst genutzten Arten Vogel-Kirsche, Pfirsich (P. persica), Pflaume (P. domestica), Aprikose (P. armeniaca), Sauer-Kirsche (P. cerasus), Mandel ( $P$. dulcis) und Schlehe ( $P$. spinosa) gehören zur Unterfamilie der Prunoideae. Aus der wilden Vogel-Kirsche sind zahlreiche Kulturformen wie die Herz-Kirsche (P. avium ssp. juliana) mit weichem Fruchtfleisch sowie die Knorpel-Kirsche (P. avium ssp. duracina) mit festem Fruchtfleisch hervorgegangen. Diese Selektionen wurden bereits mehrere Jahrhunderte v. Chr. im Schwarzmeer-Raum angebaut. Durch die Römer ge-

Abb. 1: Blühende Süßkirsche (Mitte). langten diese später auch nach Mitteleuropa. Prunus avium ist nach verschiedenen Autoren neben der Steppen-Kirsche (P. fruticosa) eine der beiden Elternarten der Sauer-Kirsche.

\section{Verbreitung und Lebensraum}

Die Vogel-Kirsche ist eine europäisch-westasiatische Baumart. Ursprünglich fehlte sie in Großteilen der norddeutschen Tiefebene, ist aber infolge zahlreicher Anpflanzungen und Verwilderungen dort mittlerweile allgemein verbreitet. Ihre Häufigkeit nimmt in Richtung Alpen deutlich zu (KiermeIER 1993). Sie ist ein typisches Element artenreicher Mischwälder, in denen sie zusammen mit Rot-Buche (Fagus sylvatica), Hainbuche (Carpinus betulus), Stiel-Eiche (Quercus robur) und Weißdorn-Arten (Crataegus spp.) vergesellschaftet ist (KIERMEIER 1995). In Hartholzauen außerhalb des regelmäßigen Überschwemmungsbereiches mit Esche (Fraxinus

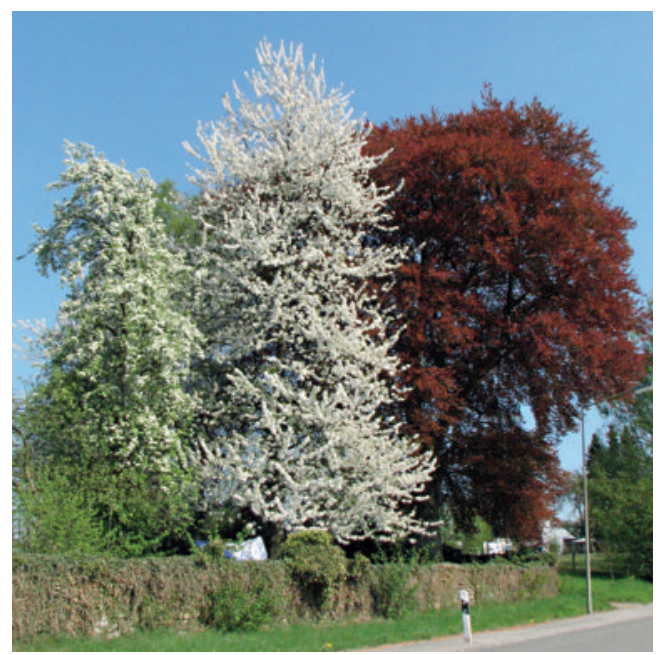



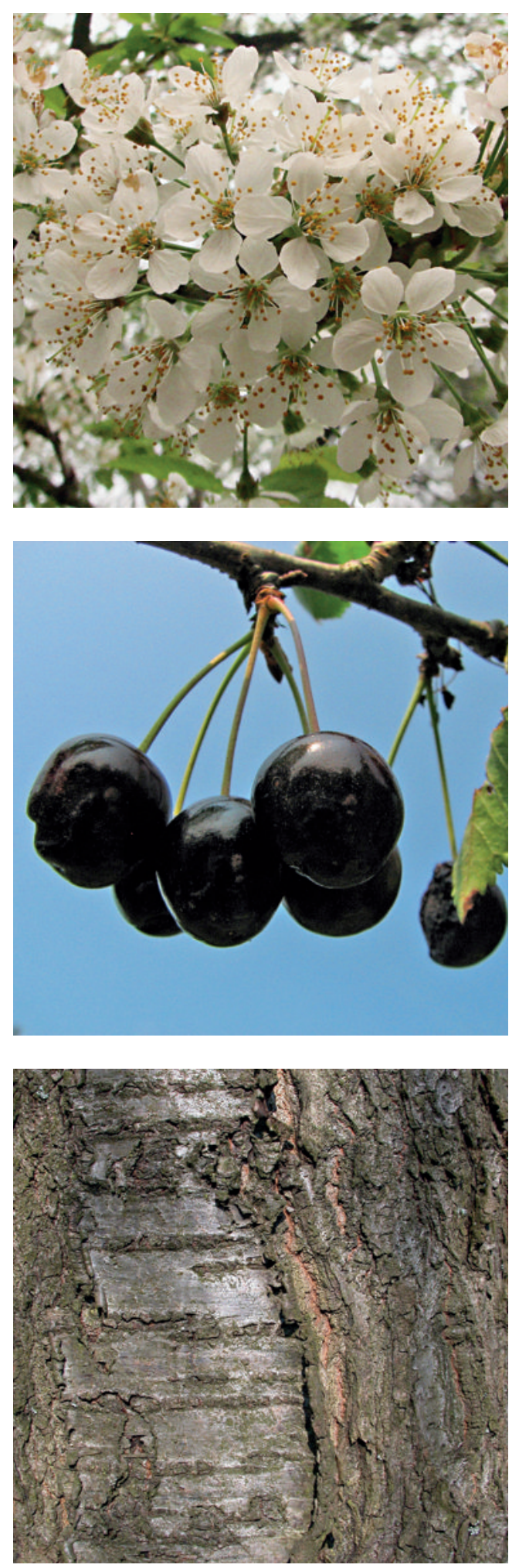

excelsior) oder Pfaffenhütchen (Euonymus europaeus) ist sie seltener, da sie empfindlich gegenüber Staunässe reagiert. Die Vogel-Kirsche gilt als Zeigerpflanze für nährstoffreiche Böden und ist besonders häufig an sonnig-warmen Standorten anzutreffen. Sie kommt daher in Mittel-Europa auch entlang von Waldrändern und Feldrainen, auf Lichtungen und in Feldgehölzen vor, wobei sie niemals größere Bestände bildet. Da die Vogel-Kirsche häufig gepflanzt wird, sind echte Wildvorkommen häufig nicht mehr als solche zu erkennen. Ihre Bestände mischen sich gelegentlich auch mit der morphologisch recht ähnlichen Sauer-Kirsche. Bei tiefen Temperaturen und mechanischen Verletzungen reagiert die Vogel-Kirsche mit einem Austreten von bernsteinfarbenem Harz, trotzdem ist sie gut winterhart. Allerdings reagiert sie gegenüber Salz empfindlich und zeigt daher in Bereichen, in denen im Winter Auftausalze verwendet werden, eine deutlich verringerte Vitalität. Ihre Industriefestigkeit ist ebenfalls nur als mäßig zu betrachten.

\section{4. Äußeres Escheinungsbild, Blüten und Früchte}

Vogel-Kirschen werden 20-25 m hoch und meist 5-8 $\mathrm{m}$ breit und damit größer als Sauer-Kirschen. Ihre Krone ist zunächst locker eiförmig, im Alter rund mit einem vergleichsweise recht kurzen Stamm. Mit einer Lebenserwartung von 80-100 Jahren werden die Bäume nicht besonders alt. Im Unterschied zur Sauer-Kirsche, bei der die Triebe recht dünn und teilweise leicht überhängend sind, sind die Äste der Vogel-Kirsche tendenziell eher steifer und sparriger verzweigt. Es gibt eine deutliche Differenzierung in Lang- und Kurztriebe. Ein Großteil der Kirschen-Verwandten enthält in den vegetativen Organen hohe Konzentrationen an Blausäure. Bei Verletzungen der Triebe verströmen diese einen intensiven Bittermandelgeruch, der durch das toxische Blausäureglykosid Prunasin hervorgerufen wird. Solche Blausäureglykoside schützen den Baum vor dem

Abb. 2 (oben): Blüten im Detail.

Abb. 3 (Mitte): Reife Früchte von Prunus avium.

Abb. 4 (unten): Stamm einer Vogelkirsche. 
Befall durch parasitische Pilze (Düll \& KUTZELNIGG 2005). Die zunächst graue, teilweise glänzende Ringelborke ist im Alter dunkelbraun gefärbt und löst sich in kurzen, krausen Streifen vom Stamm. Dieser sowie kräftigere Zweige tragen große braune Lentizellen, die dem Gasaustausch dienen und auch an alten Exemplaren erhalten bleiben.

Das zunächst rötliche, feinstrukturierte Holz der Vogel-Kirsche dunkelt im Laufe der Zeit an der Luft und im Licht nach, so dass es letztlich dunkel rotbraun und mahagoniartig wirkt. Charakteristisch sind kleine lokale Verfärbungen und vereinzelte Harzgänge. Aufgrund der leichten Bearbeitbarkeit und der attraktiven Färbung wird das Holz im Möbelbau sowie in der Tischlerei geschätzt (BEAZLEY 1976).

Als Herzwurzler entwickelt die Vogel-Kirsche einen besonders hohen Anteil an Faserwurzeln im oberen Bodenhorizont. Gelegentlich werden Ausläufer gebildet, die besonders nach Verletzungen der Wurzel in ausreichend lockeren Substraten auftreten. Diese Wurzelausläufer können Längen von bis zu 12 m erreichen.

Die wechselständigen, länglich eiförmigen und scharf gesägten Blätter sind im Austrieb bronzefarben und vergrünen rasch. Am Blattgrund sitzen zwei stark eingeschnittene, grünlichrote Nebenblätter. Unmittelbar unterhalb der Blattspreite befinden sich am rötlich überlaufenen Blattstiel grünlich-rote, warzige Verdickungen. Hierbei handelt es sich um Nektardrüsen. Da sie nicht in der Blüte ausgebildet sind, werden sie extraflorale Nektarien genannt. Sie produzieren besonders in den ersten Wochen nach dem Austrieb viel Nektar und locken somit zahlreiche Ameisen (Formica obscuripens) an, die im Gegenzug den Baum vor Schädlingen wie Raupen schützen (DÜll \& KuTZELNIGG 2005). Bei der Sauer-Kirsche fehlen diese Nektarien häufig. Die Herbstfärbung ab Mitte Oktober ist spektakulär und deckt ein breites Farbspektrum von gelb über orange bis hin zu leuchtend rot ab. Die eiförmigen, spitz zulaufenden, rotbraunen Winterknospen sind 4-5 mm lang.

Abb. 5: Das typisch orangerote Kirschholz.

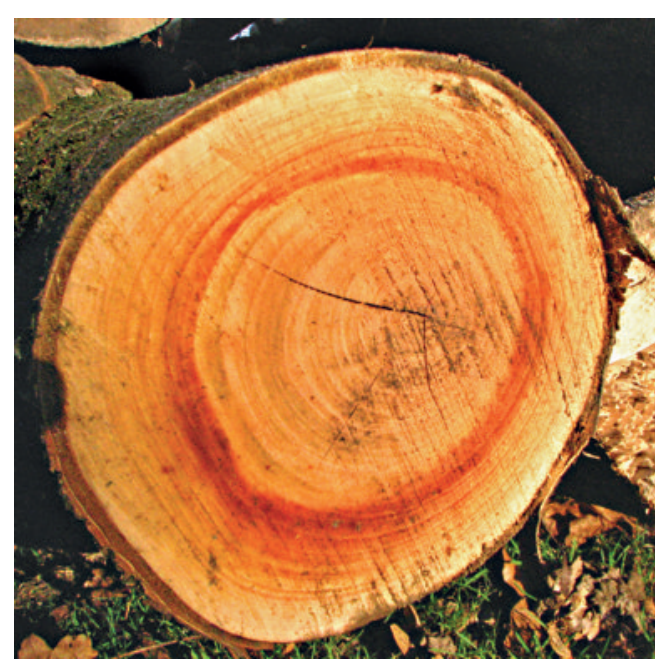

Die Blüten der Vogel-Kirsche erscheinen an ein- oder mehrjährigen Kurztrieben (sog. „Infloreszenz-Kurztriebe") mit oder während des Laubaustriebs im April noch deutlich vor der Blüte der Sauer-Kirschen. Sie verströmen einen süßlichen Duft. Beim Rückschnitt von Süß-Kirschen sollte deshalb darauf geachtet werden, dass die blütenbildenden Kurztriebe erhalten bleiben, um einen Fruchtansatz in der nächsten Saison zu gewährleisten. Nach einem starken Rückschnitt kann es zum langtriebartigen Durchwachsen der älteren Kurztriebe kommen. Im Frühjahr ist die Vogel-Kirsche mit ihren nektarreichen, reinweißen Blüten eine wichtige Bienenweide. Die fünfzählige Blüte enthält zahlreiche Staubblätter und ein Fruchtblatt, aus dem später die Kirsche entsteht.

Kirschen sind Steinfrüchte mit einem häutigen Exokarp (Schale), einem fleischigen Mesokarp (Fruchtfleisch) und einem stark verholzten Endokarp (Stein). Die rundlichen bis kugeligen Kirschen werden während der Reife leuchtend rot bis schwarzrot. Die Kerne sind für den Menschen giftig, da sie das Blausäureglykosid Amygdalin enthalten (DülL \& KUTZELNIGG 2005). Der Kernbeißer ist einer der wenigen heimischen Vogelarten, die die harten Kirschkerne knacken können, um sie später zu verzehren. Die Kirschen schmecken süßlich und sind für viele Vögel eine willkommene Nahrung. Sie scheiden die Kerne an anderer Stelle wieder aus und tragen 


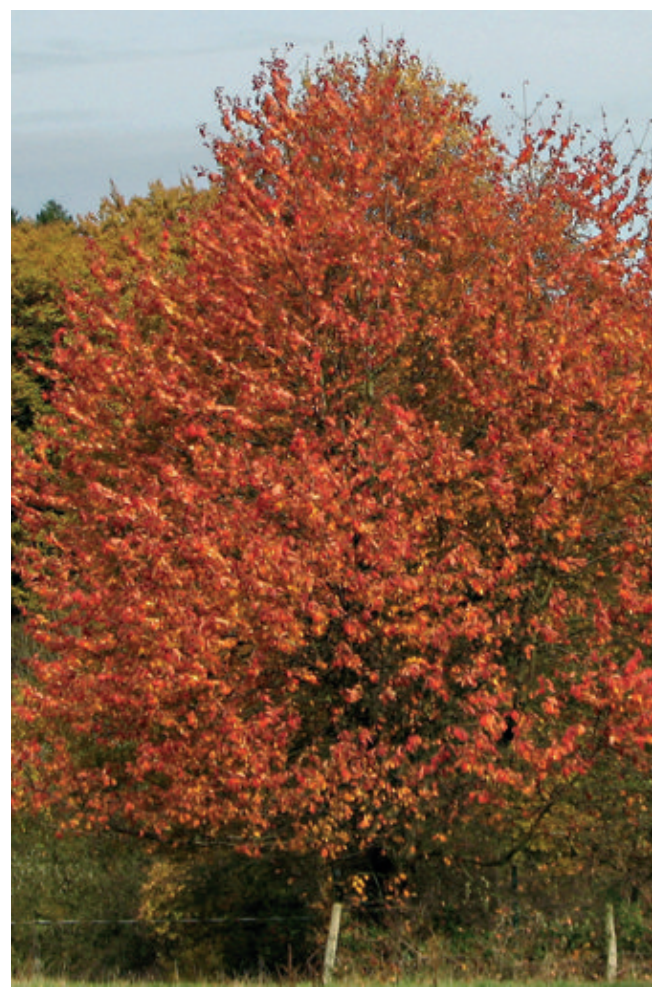

somit zur Ausbreitung bei. Auch für Nager sind die Samen in den Kernen ein gutes Futter. Zahlreiche der z. B. von Mäusen für den Winter versteckten und nicht verzehrten Samen keimen im Frühjahr aus. Wilde Süß-Kirsch-Früchte enthalten, verglichen zu den im Obstbau angepflanzten Züchtungen, einen im Vergleich zum Fruchtfleisch sehr großen Steinkern und sind insgesamt deutlich kleiner. Sauer-Kirschen haben einen rund doppelt so hohen Fruchtsäuregehalt (überwiegend Äpfelsäure) wie Süß-Kirschen und schmecken daher entsprechend weniger süß.

\section{Verwendung}

Kirschen schmecken nicht nur diversen Tieren. Prunus avium ist ein wichtiges Obstgehölz. Die Kirschen wilder sowie Kultur-Vogel-Kirschen eignen sich als Frischobst sowie zur Herstellung von Säften, Marmeladen und Gelees. Mit den Steinkernen werden, ähnlich wie mit Getreidekörnern, Kissen gefüllt, die erhitzt zur Wärmebehandlung z. B. von verspannten Muskeln eingesetzt werden. Kirschen sind nur bedingt lagerungsfähig und werden deshalb meist vor Ort verarbeitet. Hauptproduzenten für KulturKirschen sind vor allem Deutschland, die USA, Türkei und Italien (LIEBEREI \& REISDORFF 2007).

Ingenieurbiologisch eignen sich Vogel-Kirschen aufgrund ihres dichten Wurzelsystems zur Sicherung von Böschungen. Siedlungsgeschichtlich ist die Vogel-Kirsche ein wichtiger Dorf- und Hofbaum. Auch in Parkanlagen sind VogelKirschen wichtige Solitärgehölze. Im Siedlungsbereich sollte jedoch darauf geachtet werden, dass ausreichend Abstand zu Wegen, Plätzen oder Sitzgelegenheiten gewährleistet wird, da es durch herabfallende Früchte zu starker Verschmutzung kommen kann. Zudem locken herabgefallene Früchte massenhaft Wespen an. Daher empfiehlt es sich, in stark frequentierten Bereichen die gärtnerische Selektion Prunus avium 'Plena' zu pflanzen. Diese gefüllt-blühende Sorte entwickelt keine Früchte. Allerdings sind derartige Sorten tierökologisch von nur geringem Wert. Interessante Farbkontraste ergeben sich während der Blütezeit zwischen den weißen Blüten der VogelKirsche mit dunkellaubigen Gehölzen z. B. der Blut-Buche (Fagus sylvatica 'Atropurpurea').

\section{Literatur}

Roloff, A. \& BÄRTELS, A. 1996: Gartenflora

Band 1, Gehölze. - Stuttgart.

BEAZLEY, M. 1976: The international book of wood. London.

Düll, R. \& Kutzelnigg, H. 2005: Taschenlexikon der Pflanzen Deutschlands. Ein botanisch-ökologischer Exkursionsbegleiter. 6. Aufl. - Wiebelsheim.

Kiermeier, P. 1993: BdB-Handbuch, Teil VIII,

Wildgehölze des mitteleuropäischen Raumes, 5. Aufl. Pinneberg.

KiermeIER, P. 1995: Lebensbereiche der Gehölze, eingeteilt nach dem Kennziffersystem, 3. Aufl. - Pinneberg. Lieberei, R. \& REISDORFF, C. 2007: Nutzpflanzenkunde.

7. Aufl. - Stuttgart.

STÜTZEL, T. 2005: Botanische Bestimmungsübungen, praktische Einführung in die Pflanzenbestimmung, 2. Aufl. - Stuttgart.

Abb. 6: Herbstfärbung der Vogelkirsche. 